Pasado y Memoria

ISSN: 2386-4745

Núm. 24, 2022, pp. 342-355

https://doi.org/10.14198/PASADO2022.24.14

Nota bibliográfica

\title{
Un nuevo objeto histórico. La democracia y los historiadores
}

\section{A new historical subject. Democracy and the historians}

\author{
Carlos Domper Lasús \\ Universidad de Zaragoza, España \\ cdomper@unizar.es \\ https://orcid.org/0000-0001-6096-2103
}

Recibido: 29/09/2021

Aceptado: 16/11/2021

Cómo citar esta nota bibliográfica: DOMPER LASÚS, Carlos (2022). Un nuevo objeto histórico. La democracia y los historiadores. Pasado y Memoria. Revista de Historia Contemporánea, (24), pp. 342-355, https://doi.org/10.14198/PASADO2022.24.14

\section{Resumen}

A lo largo de la última década han aparecido una enorme cantidad de publicaciones dirigidas a explicar las razones por las que la democracia liberal está en crisis. Consciente o inconscientemente, muchos de esos trabajos se articulan alrededor de un concepto de democracia liberal monolítico y ahistórico que ha sido desarrollado por las ciencias sociales desde mediados de los años cincuenta. Este artículo da cuenta de los trabajos sobre la democracia que los historiadores han desarrollado desde comienzos del nuevo milenio. De este modo, muestra una visión mucho más conflictiva y problemática de este objeto de estudio anclada, en su inserción en los procesos de cambio social y político.

Palabras clave: Democracia; Historia; Ciencia Política; Crisis; Adaptación.

\begin{abstract}
Over the last decade, a huge number of works have been published, aimed at explaining the reasons behind the current crisis of liberal democracy. Whether consciously or unconsciously, many of these works are constructed on the basis of a monolithic, unhistorical concept of liberal democracy, which has been developed by the social
\end{abstract}


sciences since the mid-1950s. This article accounts for the research on democracy that historians have conducted since the beginning of the new millennium. Thus, it shows a much more conflictive and problematic approach to this subject by inserting it within processes of social and political change.

Keywords: Democracy; History; Political Science; Crisis; Adaptation.

Financiación: El autor es contratado Juan de la Cierva (Formación) en el citado departamento. Este trabajo se enmarca dentro del proyecto de investigación «La España de Franco, el legado de los fascismos y el debate sobre los contenidos de la democracia en Italia, Alemania, Bélgica y Francia (1945-1968)»(PID 2020-112800-GB).

En diciembre de 2020, la periodista Laure Mandeville realizó una larga entrevista al conocido politólogo Francis Fukuyama en el diario Le Figaro (Mandeville: 2020). De manera general, la periodista interrogó al entrevistado acerca del impacto que la pandemia provocada por el virus COVID-19 tendría para la democracia liberal. Fukuyama trató de responder con la mayor concreción posible a importantes cuestiones relacionadas con el presente inmediato a la vez que alentaba a los países occidentales a no perder la fe en la democracia liberal, frente a las soluciones propuestas por China para hacer frente a la crisis. Sin embargo, vista en perspectiva, la entrevista no deja de ser un intento de quien preconizó el fin de la historia, como consecuencia de la imposición de la democracia liberal como único régimen político viable, de defender sus tesis en un contexto en el que las mismas parecen claramente cuestionadas (Fukuyama: 1989; 1992).

A decir verdad, si tuviésemos que describir el zeitgeist de nuestra época no sería descabellado afirmar que una de sus principales características es la sensación de que el modelo de democracia que Fukuyama proclamó triunfante a finales de los ochenta se encuentra en aprietos. De hecho, a medida que la larga duración de la crisis económica iniciada en 2008 ha ido generando consecuencias políticas, en forma de aparición de alternativas iliberales a la gestión del estado y la sociedad, en países europeos, especialmente Polonia y Hungría, e incluso en los Estados Unidos, se ha producido lo que Jan-Werner Müller ha llamado recientemente «una explosión» de publicaciones sobre la «crisis de la democracia liberal» (Müller: 2021). Ahora bien, muchos de esos trabajos diseccionan la crisis y proponen soluciones sin ni si quiera plantear una mínima reflexión sobre qué es lo que está en crisis.

Que muchos de quienes hoy escriben sobre su desmoronamiento ni tan siquiera dediquen un párrafo a reflexionar sobre qué es la democracia liberal no quiere decir que este sea un tema inexplorado. La democracia se consolidó 
como objeto de estudio académico después de la IIGM. Las ciencias sociales, especialmente la ciencia política, fueron las primeras en descubrir el potencial analítico e investigador que se escondía tras un tipo de régimen político que por aquel entonces no era hegemónico. Al calor de la política exterior estadounidense que, una vez terminada la guerra, pretendía expandir la democracia liberal a todos aquellos países susceptibles de caer bajo la órbita de la Unión Soviética, muchos científicos sociales que habían participado en la IIGM como analistas e incluso como gestores sobre el terreno en la Alemania ocupada comenzaron a investigar sobre la democracia (Ball: 1993; Leffler: 2010).

Esas investigaciones se realizaron con el apoyo tanto del Departamento de Estado como de fundaciones privadas vinculadas al mismo y al amparo metodológico de la incipiente revolución conductista. Dado que como hemos señalado la extensión de la democracia liberal se convirtió en uno de los pilares de la política exterior de Estados Unidos, tanto la Casa Blanca como las citadas instituciones no gubernamentales a ella vinculadas (la Fundación Rockefeller, la Fundación Ford o la Fundación Carnegie) no dudaron en financiar con ingentes cantidades de dinero la creación de nuevos departamentos universitarios dedicados a las ciencias sociales, así como las investigaciones de sus nuevos integrantes (Gilman: 2003). Estos estudios estuvieron metodológicamente anclados en el conductismo, el paradigma que mayor influencia tuvo en las ciencias sociales estadounidenses durante la posguerra. En esencia, los conductistas aspiraban a desarrollar un modelo empírico de investigación basado en las metodologías empleadas por las ciencias naturales que permitiese: validar y dar confiabilidad a las hipótesis desarrolladas por los académicos y crear teorías que permitiesen explicar la realidad observada y predecir cómo evolucionaría (Eulau: 1969).

Como consecuencia de todo ello, los trabajos sobre la democracia que surgieron de este contexto tuvieron un carácter profundamente ahistórico. De hecho, partían de la premisa de que la investigación sobre la democracia debía basarse en la observación de cómo funcionaba realmente dicho sistema político en el lugar donde, según los propios investigadores, el mismo había alcanzado su mayor grado de perfección, es decir, Estados Unidos. Partiendo de esa realidad, se trataba de sistematizar su funcionamiento a través de diferentes variables cuantificables y aplicables a las realidades sociales, políticas, económicas y culturales de otros países. Entre ellas destacaron sobremanera la participación política, el comportamiento electoral, la cultura política y cívica de los ciudadanos, así como las características psicológicas de lo que dio en llamarse homo politicus y la relación entre modernización y democracia. A nivel general, todos estos trabajos caracterizaron la democracia liberal como 
un régimen político elitista, que otorga un papel predominante al rol que representan los grupos dirigentes y a la despolitización de grandes sectores de la sociedad; competitivo, en permanente búsqueda del equilibrio entre la oferta y la demanda del mercado político en el que los partidos actúan como vendedores y los votantes como consumidores; y pluralista, en el que la sociedad se organiza en grupos de interés que pueden torcer la voluntad del estado (Almond: 1950, 1956; Almond \& Verba: 1963; Bendix \& Lipset: 1966; Berelson \& Lazarsfeld,:1954; Dahl: 1956, 1967; Downs: 1957; Lazarsfeld et al.: 1944; S. M. Lipset \& Raab: 1970; S. Lipset \& Rokkan: 1967).

Posteriormente, con el paso de los años, el fracaso de los intentos de instaurar la democracia liberal en muchos estados surgidos de los procesos de descolonización y las duras críticas que recibió la metodología conductista por su completa omisión de la Historia, llevaron a algunos científicos sociales a preguntarse por qué algunas democracias funcionaban y otras no, y a mirar al pasado en busca de respuestas. En concreto, comenzaron a analizar lo que había sucedido con las democracias que fracasaron en Europa entre la década de los veinte y la de los treinta del siglo XX. Para ello, comenzaron a prestar atención a las estructuras socioeconómicas, los legados de las guerras, el impacto disruptivo de los conflictos étnicos, las correas de transmisión que convertían las discrepancias sociales en políticas de los partidos y los parlamentos (BergSchlosser \& Mitchell: 2000; Capoccia: 2005; Luebbert: 1991). Por primera vez, estos trabajos, entre los cuales aparecieron los de algunos historiadores que analizaban el fenómeno de la extrema derecha (Osmond: 1993; Passmore: 1997; Paxton:1997), pusieron de manifiesto que el éxito de la democracia no dependía de ella misma, sino que estaba influenciado por el contexto social, mucho más de hecho que los regímenes autoritarios. Trabajos, todos ellos, influenciados de uno u otro modo por la aproximación pionera que realizaron, a finales de los años setenta, Alfred Stepan y Juan José Linz al estudio de la crisis de la democracia (Linz \& Stepan: 1978).

Con la salvedad de los análisis que acabamos de mencionar, que además no tenían como objeto de estudio la democracia sino el fracaso de los regímenes políticos de entreguerras, la historiografía ha permanecido al margen de los estudios sobre la democracia, entendida como régimen político. De hecho, si uno revisa los trabajos historiográficos sobre los regímenes políticos de la contemporaneidad descubrirá rápidamente que, como ha señalado Martin Conway, la democracia aparece en ellos, sobre todo en Europa occidental, como el régimen político por defecto. Dicho de otra manera, la democracia se concibe como el régimen político al que los estados contemporáneos regresan cuando las condiciones específicas que generan alternativas antidemocráticas 
desaparecen. Esta concepción ahistórica de la democracia moderna asume la idea de fondo presente en los trabajos desarrollados por la ciencia política anteriormente citado, es decir, la existencia de un modelo único y homogéneo de régimen democrático (Conway: 2020: 18-19). De este modo, la historia de la misma ha quedado reducida a la de su expansión gradual desde la sociedad masculina de notables del siglo XIX hasta la consolidación del sufragio universal masculino y femenino durante la segunda mitad del siglo XX.

Sin embargo, esta es una visión muy reduccionista que obvia que la democracia tal y como la conocemos no surgió de la nada, sino que ha evolucionado a través de un proceso conflictivo que ha tenido lugar en contextos políticos diversos. Como consecuencia, durante muchos años no se ha prestado atención a un hecho fundamental: la democracia, como ideal político, está enraizada en diferentes tradiciones políticas europeas tanto de derechas como de izquierdas y las divergencias entre los modelos de democracia desarrollados por las mismas han sido a menudo más visibles que las similitudes. Dicho de otro modo, la democracia no es tanto un elemento de consenso como el resultado de las disputas instrumentalizadas por los diferentes grupos políticos para avanzar en sus propósitos partidistas. De hecho, la lucha entre dichas tradiciones por imponer sus modelos específicos ha alcanzado momentos puntuales de tensión en los que las circunstancias específicas del contexto han determinado la prevalencia de unos sobre otros.

Con algunas excepciones muy limitadas (Costopoulos \& Rosanvallon: 1995; Dippel:1985; Palmer:1953), los historiadores comenzaron a interesarse por el estudio de la democracia al comienzo del nuevo milenio y, precisamente, alrededor del anterior espacio interpretativo. En concreto, han sido la historia conceptual y la historia política las que se han enfrascado en la tarea de historiar la democracia alrededor de dos debates fundamentales: el significado de la democracia y su evolución desde el siglo XIX y las narrativas sobre la misma que han desarrollado los diferentes partidos y estados a lo largo de la contemporaneidad (Müller \& Nevers: 2019).

Por lo que respecta a la historia conceptual de la democracia, los historiadores han recurrido a las tesis de Koselleck sobre la evolución del significado de los conceptos para afirmar que cualquier definición de la misma es siempre contingente y debe ser insertada en un contexto específico. Así, pretenden analizar las diferentes interpretaciones que se han dado a dicha palabra para sacar a la luz los distintos proyectos, experiencias, expectativas, visiones y tradiciones del pasado que se esconden tras cada una de ellas, con el objetivo de desarrollar una visión plural de la democracia. De este modo, la historia conceptual ha señalado en los últimos años que el concepto de democracia, 
tal y como lo conocemos hoy, adquirió sus primeros rasgos a lo largo del siglo XVIII y, especialmente, durante la Revolución Francesa (Innes \& Philp: 2018). A partir de ahí, varios trabajos han mostrado como fue transformándose en varios países, a distintos ritmos, con diversas formas y entre diferentes grupos sociales y políticos.

Estos trabajos sobre la historia conceptual de la democracia han sido fundamentales para el desarrollo del segundo de los debates a los que nos hemos referido, el de las narrativas sobre la misma. No obstante, este área de conocimiento también se ha visto notablemente impulsada por la creciente literatura sobre la historia política de la democracia. Dichas investigaciones muestran que entre finales del siglo XIX y mediados del siglo XX la democracia se convirtió en un fenómeno político y social, si bien dicho proceso no solo no se desarrolló de manera lineal, sino que además fue muy disputado (Gijsenbergh et al.: 2012; Kurunmäki: 2019; Kurunmaki \& Strang: 2010; Lavinia Anderson: 2000; Müller \& Tooze: 2015; Richter: 2017; Ziblatt: 2006 y 2017). A lo largo de ellas, muchos países en Europa y América comenzaron a desarrollar narrativas que caracterizaban su propia historia como democrática. Cada una de esas narrativas concedió diferente relevancia a aspectos como el papel y la forma del estado, la importancia de la libertad individual, los pilares sociales de la democracia, el papel que los ciudadanos debían representar en la misma, la relación entre la soberanía popular y la salvaguardia de los intereses del país, etc. Los diferentes énfasis en cada uno de estos elementos reflejan las fracturas provocadas en cada país por los diversos legados que contribuyeron al desarrollo de la cultura democrática. En este sentido, la historiografía ha señalado que el periodo de entreguerras fue posiblemente el primer momento en el que de manera transnacional y hasta cierto punto sincrónica se desarrollaron narrativas sobre la democracia (Harrington: 2016; Ihalainen: 2017; McCarthy: 2007: 2012). De hecho, incluso las dictaduras que surgieron durante dicho periodo trataron de revestirse de cierto halo democrático (Fritzsche: 1990; Jessen \& Richter: 2011; Muller: 2013).

Ambos debates, el que se articula alrededor del significado de la democracia y el que gira en torno a las narrativas sobre la misma, convergen en lo que Conway y Buchanan catalogaron como «the greatest challenge for historians of twentieth-century democracy», es decir, explicar «why a very particular parliamentary model of democracy was so successfully implanted (with a few notable exceptions) in Western Europe after 1945» (Buchanan \& Conway: 2002: 10). En este sentido, Conway ha subrayado recientemente que es todavía habitual entre los historiadores explicar la victoria de la democracia después de 1945 sobre la base de «a dictatorship of its origins». Dicho de otro modo, 
se asume que la derrota del III Reich junto con el triunfo de las potencias aliadas proporcionan un marco explicativo suficiente para justificar lo que vino después (Conway: 2020: 20). Sin embargo, desde la perspectiva de la evolución de la historia de la democracia contemporánea, el triunfo de la democracia parlamentaria tras 1945 fue algo realmente inesperado. Tal y como han mostrado los estudios a los que hemos aludido anteriormente, desde el siglo XIX se habían desarrollado en Europa diversas concepciones y narrativas de la democracia surgidas de diferentes tradiciones intelectuales y políticas que, en muchos casos, resultaban totalmente incompatibles entre sí. Por lo tanto, cuando los diferentes países liberados de Europa occidental iniciaron su proceso de reconstrucción política inmediatamente después de la derrota alemana, no existía un modelo consensuado de democracia ni tampoco una percepción compartida sobre el pasado democrático europeo.

En este sentido, desde principios del nuevo milenio la historiografía europea se ha interesado en el análisis del proceso a través del cual la democracia liberal parlamentaria acabó imponiéndose al resto de modelos disponibles a la altura de 1945. Así, Pepijn Corduwener ha analizado de manera comparada el largo y disputado proceso a través del cual las élites políticas francesas, italianas y de la República Federal Alemana lograron alcanzar un consenso sobre el modelo de democracia que compartían y se reconocieron unas a otras como actores legítimos de la vida política de sus diversos países (Corduwener: 2016 y 2017). De igual modo, estos procesos han sido abordados de manera nacional por distintos autores que han puesto el foco en diferentes aspectos de los mismos, desde las bases intelectuales de las diversas concepciones existentes sobre la democracia, hasta su imbricación en la evolución de la historia política de cada país, pasando por el estudio de sus principales protagonistas (Bauerkämper: 2014; Bedeschi: 2013; Berstein: 1999; Forner: 2016; Nord: 2019; Orsina: 2019).

Finalmente, aunque no por ello menos importante, Martin Conway, posiblemente el principal representante de esta corriente historiográfica, es quien mejor y con más precisión ha descrito y explicado el modelo de democracia parlamentaria que acabó triunfando en la Europa occidental de posguerra. De manera muy resumida, Conway ha caracterizado dicho modelo alrededor de cinco elementos: la reafirmación de la autoridad y responsabilidad del estado-nación; la supremacía en el proceso político de parlamentos elegidos por sufragio universal masculino y femenino que, sin embargo, hicieron hincapié en la gestión y el control antes que en la participación de las masas; el alejamiento de las masas de los procesos de toma de decisiones, en manos de comités técnicos creados en el interior de los parlamentos; la limitación 
y control de las estructuras de participación popular; y, finalmente, el apoyo del individualismo y las definiciones negativas de la libertad desarrolladas por intelectuales anticomunistas como Isaiah Berlin o Raymond Aron (Conway: 2002, 2004 y 2020).

Ahora bien, estos trabajos adolecen por lo general de dos de los principales problemas que han afectado a la historiografía sobre la Europa occidental posterior a 1945, en muchas ocasiones tendente a presentar la historia del continente como una evolución casi teleológica hacia una realidad pacífica, democrática y postnacional (Judt: 2007). En primer lugar, obvian la existencia de cualquier tipo de herencia o legado de carácter autoritario proveniente del periodo anterior a la guerra. Así, aunque durante los últimos años han comenzado a aparecer trabajos sobre la continuidad de la violencia tras mayo de 1945 (Lowe: 2012 y 2017; Snyder: 2010), todavía no existen trabajos específicamente centrados en analizar cuestiones relacionadas con la pervivencia del anticomunismo o el rechazo de las masas entre la población europea. Recientemente, Gavriel D. Rosenfeld ha explorado, recurriendo a la historia contrafactual, la pervivencia en la sociedad de la República Federal de Alemania de algunos elementos sociales, políticos y culturales procedentes del periodo de entreguerras (Rosenfeld: 2019). En segundo lugar, y como consecuencia de lo anterior, excluyen sistemáticamente de sus campos de análisis las dictaduras del sur de Europa que sobrevivieron a la muerte de los regímenes que inspiraron su nacimiento. En este sentido, el Franquismo y el Estado Novo portugués han sido obviados, o mencionados de manera muy indirecta y superficial, en los grandes relatos de la historia europea de la segunda mitad del siglo XX, puesto que, en cierto modo, su presencia distorsiona esa imagen conciliadora y democrática a la que acabamos de aludir. Como afirmó Mazower, tras la derrota del Eje ambos regímenes fueron vistos por los vencedores como la «resaca de un pasado ingrato que todos querían olvidar» (Mazower: 2001: 319).

Por lo tanto, nos parece que una de las vías desde las que la historiografía española puede contribuir a estos debates en curso sobre la democracia es sin duda profundizar en el concepto de «democracia orgánica» desarrollado por el régimen de Franco. Dicha denominación no solo formó parte de las estrategias utilizadas por el Franquismo en sus intentos por lograr la aceptación de la Comunidad Internacional a partir de 1945, sino que estuvo inserta en los debates que se desarrollaron en el interior del régimen sobre sus modelos de institucionalización durante todos los años sesenta. Con todo, se trata de un tema al que los historiadores no han prestado prácticamente atención. A nivel general, los pocos trabajos existentes sobre la «democracia orgánica» se han centrado en describir el carácter corporativo que el régimen trató de imprimir 
tanto a algunas de sus instituciones como a los procesos para seleccionar a parte del personal político de las mismas, especialmente los ayuntamientos y sus concejales, así como las Cortes Españolas y sus procuradores (Giménez Martínez: 2012; Marín i Corbera: 2016). Asimismo, Javier Muñoz Soro, en un estudio todavía muy inicial pero pionero, insertó los trabajos sobre la «democracia orgánica» desarrollados en el seno de la dictadura dentro de los debates teóricos que mantuvieron tanto los intelectuales franquistas como los antifranquistas para buscar un modelo de democracia alternativo al propuesto por el liberalismo (Muñoz Soro: 2011). En ese texto, el profesor Muñoz Soro apuntaba que las reflexiones sobre dicho modelo de democracia entre los intelectuales franquistas continuaron más allá del contexto de crisis internacional que vivió el Franquismo entre 1945 y 1955. De hecho, este autor señaló que las mismas se integraron en los debates que rodearon los debates en torno a cómo debía ser el régimen tras la muerte de Franco, algo que también apuntaron Carme Molinero y Pere Ysàs (Molinero \& Ysàs: 2008). Por último, Carlos Domper Lasús ha realizado recientemente una primera contextualización del concepto de «democracia orgánica» que desarrollaron el Franquismo y el Estado Novo durante el periodo constituyente que se abrió en Europa occidental tras la derrota alemana de 1945 (Domper Lasús: 2019).

Finalmente, nos gustaría concluir estas líneas señalando que a pesar de ser unos latecomers en el estudio de la democracia, el trabajo realizado por los historiadores en este ámbito permite observar las dificultades por las que atraviesa la democracia liberal desde una nueva perspectiva. Así, mientras la gran mayoría de las publicaciones sobre la crisis de la democracia que podemos leer hoy en día parten de la existencia de un modelo de gobierno de la sociedad concreto, único e invariable, que se encuentra amenazado, la historiografía nos muestra la crisis como una característica intrínseca de la democracia. De este modo, como ha señalado Martin Conway, la democracia no es un sujeto histórico monolítico que ha permanecido inalterado a lo largo de la historia. Por el contrario, cuando hablamos de democracia nos estamos refiriendo a un conjunto diverso y discontinuo de herramientas e ideas que se han utilizado para afrontar el gobierno de las comunidades sociales durante los últimos dos siglos y que albergan en su seno fuentes particulares de tensión y de conflicto. Como consecuencia de ello, el éxito de la democracia no deriva de su invariabilidad como modelo político, sino precisamente de lo contrario, es decir, de su capacidad para adaptarse a los cambios sociales, a pesar de que esa adaptabilidad conlleve necesariamente la fragilidad de sus estructuras y, por ello, de su legitimidad ante los ojos de los ciudadanos. 


\section{Bibliografía}

ALMOND, Gabriel Abraham (1950). The American people and foreign policy. Harcourt: Brace.

ALMOND, Gabriel Abraham (1956). Comparative Political Systems. The Journal of Politics, 18(3), 391-409. https://doi.org/10.2307/2127255

ALMOND, Gabriel Abraham, y VERBA, Sidney. (1963). The civic culture: Political attitudes and democracy in five nations. Princeton: Princeton University Press.

BALL, Terence. (1993). American political science in its postwar political context. En James FARR y Raymond SEIDELMAN (Eds.), Discipline and history. Political science in the United States (207-221). Ann Arbor: The University of Michigan Press.

BAUERKÄMPER, Arnd. (2014). The Twisted Road to Democracy as a Quest for Security: Germany in the Twentieth Century. German History, 32(3), 431-455. https://doi.org/10.1093/gerhis/ghu066

BEDESCHI, Giuseppe. (2013). La prima Repubblica (1946-1993). Storia di una democrazia difficile. Soveria Mannelli: Rubbettino.

BENDIX, Reinhard., y LIPSET, Seymur Martin (1966). Class, status, and power: Social stratification in comparative perspective. Michigan: Free Press.

BERELSON, Bernad, y LAZARSFELD, Paul Felix (1954). Voting: A study of opinion formation in a presidential campaign. Chicago: University of Chicago Press.

BERG-SCHLOSSER, Dirk, y MITCHELL, Jeremy (Eds.). (2000). The Conditions of Democracy in Europe 1919-39: Systematic Case Studies. New York: Palgrave Macmillan. https://doi.org/10.1057/9780333993774

BERSTEIN, Serge (1999). De la démocratie plébiscitaire au gaullisme: Naissance d'une nouvelle culture politique républicain. En Serge BERSTEIN (Coord.), Les cultures politiques en France (153-187). Paris: Editions du Seuil.

BUCHANAN, Tom., y CONWAY, Martin. (2002). The Politics of Democracy in Twentieth-Century Europe: Introduction. European History Quarterly, 32, 7-12. https://doi.org/10.1177/0269142002032001559

CAPOCCIA, Giovanni (2005). Defending democracy: Reactions to extremism in interwar Europe. Baltimore: Johns Hopkins University Press. https://doi.org/1 0.1080/03612759.2005.10526668

CONWAY, Martin (2002). Democracy in Postwar Western Europe: The triumph of a political model. European History Quarterly, 32(1), 59-84.

CONWAY, Martin (2004). The rise and fall of Western Europe's democratic age, 1945-1973. Contemporary European History, 13(1), 67-88.

CONWAY, Martin (2020). Western Europe's Democratic Age: 1945-1968. Princeton: Princeton University Press. 
CORDUWENER, Pepijn (2016). Democracy as a contested concept in Post-war Western Europe: A comparative study of political debates in France, West Germany and Italy. Historical Journal, 59(1), 197-220.

CORDUWENER, Pepijn (2017). The problem of democracy in postwar Europe: Political actors and the formation of the postwar model of democracy in France, West Germany and Italy. New York: Routledge.

COSTOPOULOS, Philip J., y ROSANVALLON, Pierre (1995). The History of the Word «Democracy» in France. Journal of Democracy, 6(4), 140-154. https:// doi.org/10.1353/jod.1995.0072

DAHL, Robert Alan (1956). A preface to democratic theory: How does popular sovereignty function in America? Chicago: University of Chicago Press.

DAHL, Robert Alan (1967). Pluralist democracy in the United States: Conflict and consent. Chicago: Rand McNally.

DIPPEL, Horst (1985). Democratie, Democrates. En Handbuch politisch-sozialer grundbergriffe in frankreich, 1680-1820 (pp. 57-97). Oldenbourg.

DOMPER LASÚS, Carlos (2019). Ni liberales ni comunistas. La «democracia orgánica» y la integración del Franquismo y el Estado Novo en la Europa posterior a 1945. Espacio Tiempo y Forma. Serie V, Historia Contemporánea, 31, 151-172. https://doi.org/10.5944/etfv.31.2019.24281

DOWNS, Anthony (1957). An economic theory of democracy. New York: HarperCollins.

EULAU, Heinz (1969). Behavioralism in Political Science. New York: Atherton Press

FORNER, Sean A. (2016). German Intellectuals and the Challenge of Democratic Renewal: Culture and Politics after 1945. Cambridge: Cambridge University Press

FRITZSCHE, Peter (1990). Rehearsals for Fascism: Populism and Political Mobilization in Weimar Germany. Oxford: Oxford University Press.

FUKUYAMA, Francis (1989). The End of History. The National Interest, 16, 3-18.

FUKUYAMA, Francis (1992). The End of History and the Last Man. New York: The Free Press.

GIJSENBERGH, Joris, DE JONG, Wim, HOLLANDER, Saskia, y HOUWEN, Tim (2012). Creative Crises of Democracy. Bruselas: Presses Interuniversitaires Europeennes.

GILMAN, Nils (2003). Mandarins of the Future. Modernization theory in Cold War America. Baltimore: Johns Hopkins University Press Books.

GIMÉNEZ MARTÍNEZ, Miguel Ángel (2012). Las Cortes Españolas en el régimen de Franco: Nacimiento, desarrollo y extinción de una cámara orgánica. Madrid: Congreso de los Diputados, Departamento de Publicaciones.

HARRINGTON, Austin. (2016). German Cosmopolitan Social Thought and the Idea of the West: Voices from Weimar. Cambridge: Cambridge University Press. https://doi.org/10.1017/CBO9781316275658 
IHALAINEN, Pasi (2017). The Springs of Democracy: National and Transnational Debates on Constitutional Reform in the British, German, Swedish and Finnish Parliaments, 1917-1919. Helsinki: Suomalaisen Kirjallisuuden Seura.

INNES, Joanna, y PHILP, Mark (2018). 'Democracy' from Book to Life: The Emergence of the Term in Active Political Debate, to 1848. En Jussi KURUNMÄKI, Hendrik TE VELDE y Jeppe NEVERS (Eds.) Democracy in Modern Europe: A Conceptual History (16-41). New York/Oxford: Berghahn Books.

JESSEN, Ralph y RICHTER, Hedwig (Eds.) (2011). Voting for Hitler and Stalin Elections under 20th Century Dictatorships. Frankfurt: Campus Verlag.

JUDT, Tony (2007). Postwar: A history of Europe since 1945. London: Pimlico.

KURUNMÄKI, Jussi (2019). Democracy both young and old: Finland, Sweden and the interwar crisis of democracy. Journal of Modern European History, 17(4), 486-499. https://doi.org/10.1177/1611894419880461

KURUNMAKI, Jussi, y STRANG, Johan (2010). Rhetorics of Nordic Democracy. Helsinki: Suomalaisen Kirjallisuuden Seura.

LAVINIA ANDERSON, Margaret (2000). Practicing democracy: Elections and political culture in Imperial germany. Princeton: Princeton University Press.

LAZARSFELD, Paul Felix, BERELSON, Bernard, y GAUDET, Hazel (1944). The people's choice: How the voter makes up his mind in a presidential campaign. New York: Duell, Sloan and Pearce.

LEFFLER, Melvyn Paul (2010). The emergence of an American grand strategy, 1945-1952. En M. P. LEFFLER y O. A. WESTAD (Eds.). The Cambridge history of the Cold War. Vol. I Origins (67-88). Cambridge: Cambridge University Press.

LINZ, Juan José, y STEPAN, Alfred (1978). The Breakdown of Democratic Regimes. Baltimore: The Johns Hopkins University Press.

LIPSET, Seymour Martin, y RAAB, Earl (1970). The politics of unreason: Right-wing extremism in America, 1790-1970. New York: Harper \& Row.

LIPSET, Seymour Martin, y ROKKAN, Stein (Eds.). (1967). Party systems and voter alignments. Cross national perspectives. New York: Free Press/Collier Macmillian.

LOWE, Keith (2012). Savage Continent: Europe in the Aftermath of World War II. London: Viking.

LOWE, Keith (2017). El miedo y la libertad: Cómo nos cambió la Segunda Guerra Mundial. Barcelona: Galaxia Gutemberg.

LUEBBERT, Gregory M. (1991). Liberalism, Fascism, or Social Democracy: Social Classes and the Political Origins of Regimes in Interwar Europe. Oxford: Oxford University Press.

MANDEVILLE, Laure (2020). Un long combat commence avec la Chine, mais nous n'allons pas nécessairement le perdre, Le Figaro, 26 de diciembre.https://www.lefigaro.fr/vox/societe/francis-fukuyama-un-long-combat- 
comme-avec-la-chine-mais-nous-n-allons-pas-necessairement-le-perdre20201226

MARÍN I CORBERA, Martí (2016). La democracia orgánica como forma de representación política: Un análisis de las fórmulas de cooptación de procuradores en Cortes y de sus resultados. 16, 89-112.

MAZOWER, Mark (2001). La Europa negra: Desde la Gran Guerra hasta la caída del comunismo. Barcelona: Ediciones B.

MCCARTHY, H. (2007). Parties, voluntary associations, and democratic politics in interwar Britain. The Historical Journal, 50(4), 891-912. https://doi. org/10.1017/S0018246X07006425

MCCARTHY, Helen (2012). Whose democracy? Histories of British political culture between the wars. The Historical Journal, 55(1), 221-238.

MOLINERO, Carme e YSÀS, Pere (2008). La anatomia del franquismo. De la supervivencia a la agonia, 1945-1977. Barcelona: Crítica.

MÜLLER, Jan-Werner (2021). What's Behind the «Crisis of Democracy»?, Project Syndicate, https://www.project-syndicate.org/onpoint/crisis-of-democracybooks-how-to-fix-the-system-jan-werner-mueller-2021-08

MÜLLER, Jan-Werner (2013). Contesting Democracy: Political Ideas in TwentiethCentury Europe. New Haven: Yale University Press.

MÜLLER, Tim B., y NEVERS, J. (2019). Narratives of democracy: A call for historical studies. Journal of Modern European History, 17(2), 123-134. https://doi. org/10.1177/1611894419835739

MÜLLER, Tim B., y TOOZE, Adam (2015). Normalität und Fragilität: Demokratie nach dem Ersten Weltkrieg. Hamburg: Hamburguer Edition.

MUÑOZ SORO, Javier (2011). Los apellidos de la democracia. Los intelectuales y la idea de democracia durante el franquismo (1939-1975). Cercles: revista d'història cultural, 14, 55-81.

NORD, Philip (2019). Narratives of democracy in post-war France. Journal of Modern European History, 17(2), 209-219. https://doi.org/10.1177/1611894419835751

ORSINA, Giovanni (2019). Party democracy and its enemies: Italy, 19451992. Journal of Modern European History, 17(2), 220-233. https://doi. org/10.1177/1611894419835752

OSMOND, Jonathan (1993). Rural Protest in the Weimar Republic: The Free Peasantry in the Rhineland and Bavaria. New York: Palgrave Macmillan. https:// doi.org/10.1007/978-0-333-52448-0

PALMER, Robert Roswell (1953). Notes on the Use of the Word «Democracy» 1789-1799. Political Science Quarterly, 68(2), 203-226. https://doi. org/10.2307/2144967

PASSMORE, Kevin (1997). From Liberalism to Fascism: The Right in a French Province, 1928-1939. Cambridge: Cambridge University Press. https://doi. org/10.1017/СBO9780511582103 
PAXTON, Robert (1997). French Peasant Fascism: Henry Dorgères' Greenshirts and the Crises of French Agriculture, 1929-1939. Oxford: Oxford University Press.

RICHTER, Hedwig (2017). Moderne Wahlen: Eine Geschichte der Demokratie in Preußen und den USA im 19. Jahrhundert. Hamburg: Hamburger Edition.

ROSENFELD, Gavriel David (2019). The Fourth Reich: The Specter of Nazism from World War II to the Present. Cambridge: Cambridge University Press.

SNYDER, Timothy (2010). Bloodlands: Europe Between Hitler and Stalin. New York: Basic Books.

ZIBLATT, Daniel (2006). How Did Europe Democratize? World Politics, 58(2), 311-338. https://doi.org/10.1353/wp.2006.0028

ZIBLATT, Daniel (2017). Conservative Parties and the Birth of Democracy. Cambridge: Cambridge University Press. https://doi.org/10.1017/9781139030335 